\title{
Agua potable como posible fuente de brote de diarrea por Microsporidium spp. en pacientes inmunocomprometidos en un hospital pediátrico
}

\author{
Paulina Coria, Claudia Urízar, Andrea Alba, Isabel Noemí, Anita Pino y José Luis Cerva
}

The water supply of a pediatric hospital as a possible source of an outbreak of diarrhea due to Microsporidium spp. in immunocompromised patients

Introduction: The hospital water supply is a reservoir of a variety of potentially pathogenic microorganisms that can particularly affect children and immunocompromised patients. Potentially pathogenic Microsporidium spp. have been identified in water. Microsporidiosis is an emerging parasitic and opportunistic infection in immunocompromised patients. Objective and Method: to describe an outbreak of nosocomial diarrhea due to Microsporidium, species Encephalitozoon intestinalis. Results: Seven cases of E. intestinalis associated diarrhea were reported between november 2012 and february 2013, in a unit of immunocompromised patients in L. Calvo Mackenna Children's Hospital. Microsporidium spp. was found in the hospital water supply and water reservoir tank. Secondary cases were transmitted by contact. Control measures included contact precautions, not to use faucet water for hand washing, bottled water for drinking and water reservoir tank sanitation. Conclusions: This research is about a nosocomial outbreak associated with water supply. Water quality in Chilean hospitals is an unresolved issue, especially in immunocompromised patient areas. Compliance of cleaning and disinfection of water supply systems in hospitals must be ensured.

Key words: Microsporidium spp., Encephalitozoon intestinalis, water supply, healthcare-associated infections. Palabras clave: Microsporidium spp., Encephalitozoon intestinalis, agua potable, infecciones nosocomiales.

\section{Introducción}

$\mathrm{E}$ 1 ambiente hospitalario es una fuente frecuente de exposición a numerosos y variados agentes infecciosos, los que se transmiten a través del aire, agua y por contacto ${ }^{1}$.

Los sistemas de suministro de agua potable de los recintos hospitalarios albergan una gran variedad de microorganismos, los cuales pueden encontrarse como biopelículas en estanques, sedimento y en el sistema de cañerías y grifos ${ }^{2-4}$.

Se ha descrito la capacidad de algunos microorganismos de sobrevivir en los estanques de almacenamiento de agua y existen numerosas publicaciones que han identificado al agua como el origen de infecciones y de brotes intrahospitalarios ${ }^{2-7}$.

Dentro de los agentes patógenos presentes en el agua se encuentran bacilos gramnegativos (Pseudomonas aeruginosa, Acinetobacter spp., Stenotrophomonas maltophilia, Legionella pneumophila), micobacterias no tuberculosas, hongos (Aspergillus spp., Fusarium spp.), virus (norovirus) y parásitos (Cryptosporidium spp., Microsporidium spp. $)^{8-11}$.

Los mecanismos de transmisión de infecciones por microorganismos presentes en el agua en las instituciones de salud pueden ser por contacto directo (hidro o baneoterapia) o indirecto por material re-esterilizado, ingestión de agua o hielo, inhalación de aerosoles originados por fuentes de agua, etc. ${ }^{1,4,8,9}$.

Los niños en general, y los pacientes inmunocomprometidos, tienen un mayor riesgo de desarrollar infecciones nosocomiales por patógenos presentes en el agua ${ }^{5,10,11}$.

Se describe a continuación las características epidemiológicas de un brote de diarrea nosocomial en una unidad de pacientes inmunocomprometidos causada por Microsporidium de la especie Encephalitozoon intestinalis.

Dentro del estudio del brote se encontró presencia de Microsporidium spp. en el agua de los estanques y de la salida de los grifos.

\section{Pacientes y Método}

Estudio descriptivo en base a la recolección de resultados de exámenes de deposiciones para Microsporidium spp. desde el Laboratorio de Parasitología y revisión de fichas clínicas de los pacientes internados en la Unidad de
Hospital Luis Calvo Mackenna, Santiago, Chile. Unidad de Infecciones Asociadas a la Atención de Salud (PC, CU). Unidad de Segunda Infancia (AA). Laboratorio de Parasitología (IN, AP, JLC).

Los autores declaran no tener conflictos de interés.

No hubo financiamiento.

Recibido: 29 de octubre de 2015 Aceptado: 14 de junio de 2016

Correspondencia a:

Paulina Coria De la Hoz paulina.coria72@gmail.com 
Inmunocomprometidos de Segunda Infancia del Hospital de Niños Luis Calvo Mackenna (HLCM), entre noviembre de 2011 y febrero de 2012.

Definición de caso: paciente inmunocomprometido con diarrea aguda (deposiciones líquidas y/o aumento de su frecuencia no atribuible a otra patología gastrointestinal o por fármacos), con nexo epidemiológico al caso índice y con tinción de Microsporidium spp. positiva en muestra de deposiciones.

La presencia de Microsporidium spp. en deposiciones se realizó por tinción de Cromotropo 2R (tinción de Ziehl Neelsen modificado) en el Laboratorio de Parasitología del HLCM y la confirmación e identificación de especie

Figura 1. Tinción positiva para Encephalitozoon intestinalis por técnica de inmunofluorescencia indirecta (IFI) mediante anticuerpos monoclonales. Muestra de deposiciones del caso índice.

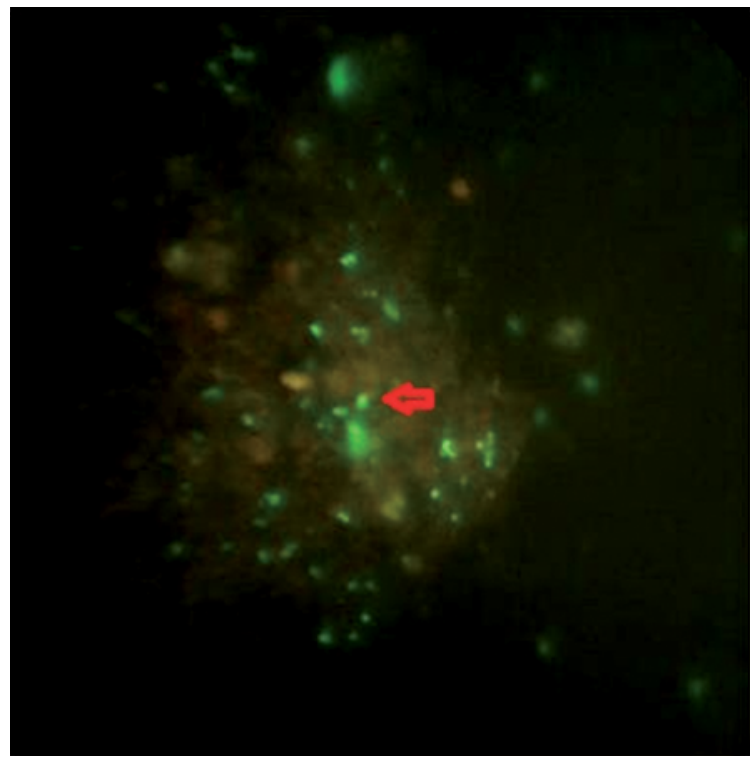

\section{Resultados}

En febrero de 2012 se observó un aumento inusual del número de casos de pacientes inmunocomprometidos con diarrea aguda y crónica con presencia de Microsporidium spp. en muestras de deposiciones en la Unidad de Segunda Infancia de nuestra institución. Por sospecha de estar frente a la existencia de un brote, se revisaron en el Laboratorio de Parasitología todos los exámenes positivos para Microsporidium spp. de pacientes internados en esa unidad desde el último trimestre del año 2011 hasta febrero de 2012. Durante este período, en el pabellón de inmunocomprometidos, se encontraron siete casos de pacientes con Microsporidium spp. positivos en deposiciones. Se confirmaron los exámenes positivos mediante IFI identificándose Microsporidium de la especie E. intestinalis en todos los casos.

Se determinó que el caso índice había sido un niño con una inmunodeficiencia congénita (síndrome de WiscottAldrich) que cursaba con una diarrea crónica y que había ingresado en noviembre de 2011 para el estudio etiológico de la diarrea. El coprocultivo y copro-parasitológico al ingreso no detectaron patógenos; sin embargo, la tinción para Microsporidium spp. resultó positiva (Figura 1). Los seis casos secundarios aparecieron en forma secuencial: un paciente receptor de un trasplante de precursores hematopoyéticos, un paciente con una leucemia mieloide aguda, dos niños receptores de trasplante hepático, otro de trasplante renal y un niño con una angiomatosis intestinal. Las características clínicas y evolución de los pacientes se describen en la Tabla 1. En todos los niños la diarrea fue acuosa o disgregada, sin elementos patológicos. Todos los pacientes recibieron tratamiento con albendazol por un mínimo de 21 días, y tuvieron buena evolución, salvo dos niños que evolucionaron con una diarrea crónica persistente. El brote afectó a siete niños, entre noviembre de 2011 y febrero de 2012, de un total de 351 pacientes internados en ese período en la unidad, lo que representó una tasa de ataque de 1,9\%. La curva epidémica correspondió a un brote de fuente común (Figura 2).

La aparición secuencial de casos secundarios hizo plantear la hipótesis de una transmisión cruzada entre los pacientes por falta en el cumplimiento de higiene

Figura 2. Curva epidémica de brote de diarrea por Microsporidium spp. Hospital L. Calvo Mackenna. 


\begin{tabular}{|c|c|c|c|c|c|c|}
\hline Caso & Edad & Diagnóstico & Inicio de la diarrea & $\begin{array}{l}\text { Fecha y exámenes de deposicio- } \\
\text { nes para Microsporidium }\end{array}$ & $\begin{array}{l}\text { Tratamiento y } \\
\text { duración }\end{array}$ & $\begin{array}{c}\text { Duración de la diarrea } \\
\text { Evolución }\end{array}$ \\
\hline $\begin{array}{l}\text { Caso } 1 \\
\text { (índice) }\end{array}$ & 1 año & $\begin{array}{c}\text { Síndrome Wiscott-Aldrich } \\
\text { Diarrea crónica }\end{array}$ & 1 de noviembre de 2011 & $\begin{array}{c}7 \text { de diciembre de } 2011 \\
\text { Microsporidium }(+++) \\
19 \text { de diciembre de } 2012(+)\end{array}$ & $\begin{array}{l}\text { Albendazol } \\
21 \text { días }\end{array}$ & $\begin{array}{l}\text { Diarrea crónica } \\
\text { persistente }\end{array}$ \\
\hline Caso 2 & 14 años & $\begin{array}{l}\text { LLA } \\
\text { TPH }\end{array}$ & 7 de enero de 2012 & $\begin{array}{l}10 \text { de enero de } 2012 \\
\text { Toxina A/B CD }(+) \\
\text { RPC ADV }(+) \\
\text { Microsporidium (+) }\end{array}$ & $\begin{array}{l}\text { Albendazol } \\
21 \text { días }\end{array}$ & $\begin{array}{l}7 \text { días } \\
\text { Buena }\end{array}$ \\
\hline Caso 3 & 11 años & LMA & 31 de enero de 2012 & $\begin{array}{l}1 \text { de febrero de } 2012 \\
\text { Rotavirus }(+) \\
\text { Microsporidium }(+)\end{array}$ & $\begin{array}{l}\text { Albendazol } \\
21 \text { días }\end{array}$ & $\begin{array}{c}5 \text { días } \\
\text { autolimitada }\end{array}$ \\
\hline Caso 4 & 2 años & Trasplante hepático & 1 de febrero de 2012 & $\begin{array}{c}3 \text { de febrero de } 2012 \\
\text { Colitis por CMV (biopsia de colon) } \\
\text { Microsporidium (+) }\end{array}$ & $\begin{array}{l}\text { Albendazol } \\
21 \text { días } \\
\text { Ganciclovir }\end{array}$ & $\begin{array}{l}10 \text { días } \\
\text { Buena }\end{array}$ \\
\hline Caso 5 & 1 año & $\begin{array}{l}\text { Angiomatosis intestinal } \\
\text { diarrea crónica }\end{array}$ & 15 de enero de 2012 & $\begin{array}{l}13 \text { de febrero de } 2012 \\
\text { Microsporidium }(+)\end{array}$ & $\begin{array}{l}\text { Albendazol } \\
21 \text { días }\end{array}$ & $\begin{array}{l}\text { Diarrea crónica } \\
\text { persistente }\end{array}$ \\
\hline Caso 6 & 2 años & Trasplante renal & 12 de febrero de 2012 & $\begin{array}{l}5 \text { de marzo de } 2012 \\
\text { Microsporidium }(+)\end{array}$ & $\begin{array}{l}\text { Albendazol } \\
21 \text { días }\end{array}$ & 7 días \\
\hline Caso 7 & 1 año & Trasplante hepático & 2 de marzo de 2012 & $\begin{array}{l}12 \text { de marzo de } 2012 \\
\text { Microsporidium }(+)\end{array}$ & $\begin{array}{l}\text { Albendazol } \\
21 \text { días }\end{array}$ & 18 días \\
\hline
\end{tabular}

LLA: leucemia linfoblástica aguda; TPH: trasplante de precursores hematopoyéticos; LMA: leucemia mieloide aguda; CD: Clostridium difficile; RPC: reacción de polimerasa en cadena; ADV: adenovirus; CMV: citomegalovirus.

de manos y de precauciones de contacto por parte del personal de salud en pacientes con factores de riesgo. El quiebre de estos procesos pudo haberse favorecido por la insuficiencia de personal de salud por vacaciones. Sin embargo, dada la aparición de casos aislados en otras unidades clínicas del hospital durante ese período, se realizó una investigación epidemiológica ambiental en búsqueda de un potencial reservorio.

Debido a las características del agente se realizó una búsqueda de Microsporidium spp. en estanques y salidas de agua del hospital, estudio que demostró la presencia abundante de Microsporidium spp., (más de 100 por campo mayor) por visualización directa en el Laboratorio de Parasitología (Figura 3) y por análisis de la calidad bacteriológica del agua del hospital solicitado a un organismo externo autorizado por el ISP.

Durante el estudio del brote se objetivó un incumplimiento de la sanitización de los estanques de agua del hospital en ese período y la inexistencia de un programa de mantenimiento del sistema de provisión de agua en la institución. Además, hasta ese momento no existía un sistema de vigilancia activa y de notificación de infecciones por parásitos no habituales en pacientes inmunocomprometidos.

Se tomaron las siguientes medidas de control de brote: se informó a las autoridades del hospital y equipo de salud, se aisló en cohorte a los pacientes afectados, se efectuó la supervisión de cumplimiento de las precauciones de contacto a todos los pacientes con pautas de cotejo que incluyeron el uso de guantes y pecheras desechables de

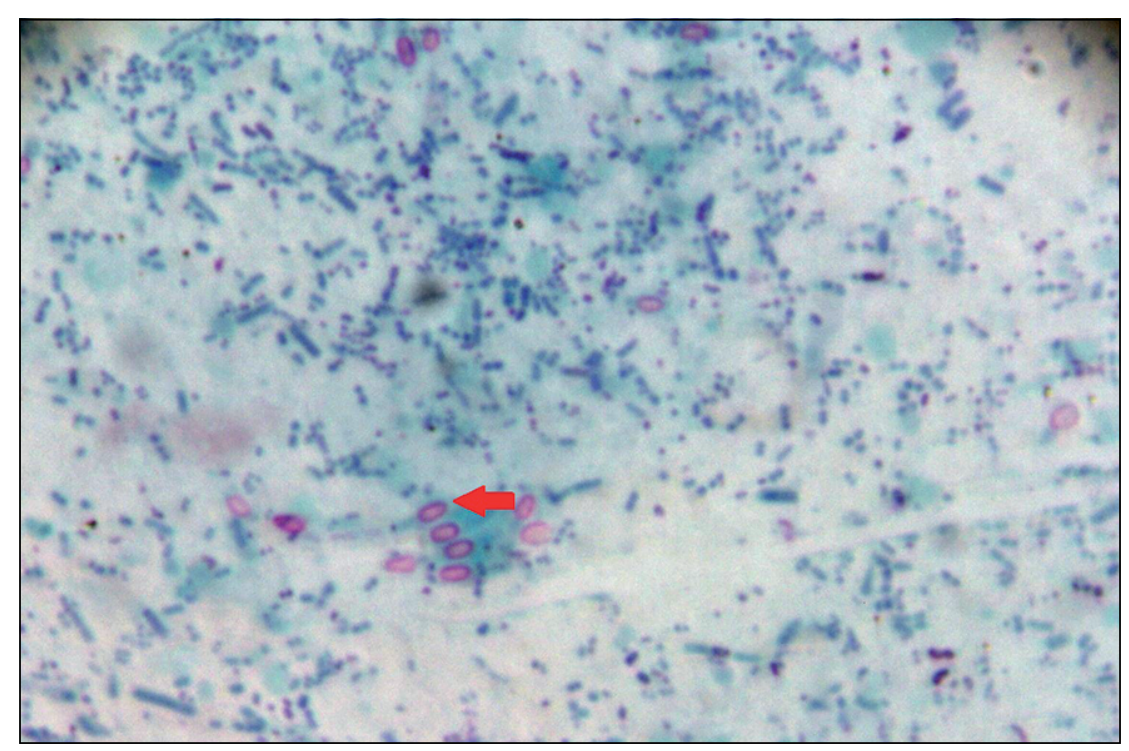

Figura 3. Presencia de formas de Microsporidium spp. en la tinción de Ziehl Neelsen modificado. Aumento 100x. Muestras de agua potable de grifo de la Unidad de Aislamiento. 
un solo uso y equipos de monitorización individuales. Además se recomendó, en forma transitoria, no usar agua de los grifos para el lavado de manos del personal y se la sustituyó por alcohol gel. Se normó el uso de agua envasada estéril para el aseo de niños inmunocomprometidos mientras no se hubiera superado la deficiencia detectada y se solicitó a las autoridades del hospital gestionar el lavado y desinfección de los estanques de agua previa medición de $\mathrm{pH}$ y cloro residual del agua del hospital. Después de efectuadas estas medidas se logró, en controles posteriores, eliminar la presencia de estos microorganismos con las metodologías ya descritas y no se presentaron nuevos casos de diarrea por Microsporidium spp. de adquisición nosocomial en un seguimiento de tres meses.

\section{Discusión}

La microsporidiosis es una infección parasitaria oportunista en pacientes inmunocomprometidos como pacientes con SIDA, receptores de trasplante de órganos, cáncer y enfermedades autoinmunes ${ }^{12,13}$.

Microsporidium spp. fue reconocido como patógeno en humanos en 1959, donde se encontró en un niño con encefalitis ${ }^{12}$. En 1985 aparecieron los primeros reportes de síndrome diarreico asociado a microsporidiosis en pacientes con infección por $\mathrm{VIH}^{14}$.

El philum Microsporidia es un grupo de parásitos intracelulares eucariontes, que contiene más de 1.000 especies distribuidas en alrededor de 150 géneros, de los cuales los que causan enfermedad en humanos son: Nosema, Pleistophora, Encephalitozoon, Enterocytozoon, Trachipleistophora, Brachiola, Anncaliia y Microsporidium. Históricamente ha sido considerado como un protozoo primitivo; sin embargo, nuevos análisis filogenéticos lo han relacionado más como un hongo.

Los microorganismos de la especie Microsporidia son ubicuos en el ambiente y puede infectar a animales vertebrados e invertebrados. Forma esporas unicelulares ovaladas, de tamaño 1,0-3,0 x 1,5-4,0 mM, muy resistentes al medio ambiente.

Alrededor de 14 especies infectan al ser humano, siendo Enterocytozoon bieneusi y Encephalitozoon intestinalis las especies más comúnmente aisladas desde el aparato digestivo ${ }^{12}$.

Las especies de Microsporidium potencialmente patógenas para el hombre han sido identificadas en el agua y en animales, adquiriendo importancia como una enfermedad zoonótica transmitida por el agua y alimentos ${ }^{13}$.

La primera confirmación de la presencia de especies de Microsporidium: E. intestinalis, E. bieneusi y Vittaforma corneae en agua, por métodos de biología molecular, fue en $1998^{15}$.

El contacto con fuentes de agua ha sido encontrado como factor de riesgo independiente para desarrollar microsporidiosis en algunos estudios ${ }^{16,17}$.

Las esporas de Encephalitozoon cuniculi permanecen viables por seis días en el agua y hasta cuatro semanas en ambiente seco a $22^{\circ} \mathrm{C}$ y las de Nosema bombycis mantienen su viabilidad durante 10 años en agua destilada ${ }^{12}$.

La mayoría de las microsporidiosis son transmitidas por la ingestión de las esporas, siendo el sitio de infección más frecuente el tracto gastrointestinal. Éstas son viables e infectantes, y están presentes en fluidos corporales (deposiciones, orina y/o secreciones respiratorias) durante la infección, pudiendo ocurrir una transmisión persona a persona.

En hospederos inmunocompetentes puede causar una enfermedad autolimitada o bien ser asintomática. En pacientes inmunocomprometidos, principalmente en pacientes con infección por VIH, está asociado a un amplio rango de manifestaciones clínicas. Puede causar desde cuadros de diarrea con síndrome de emaciación a perforación intestinal, peritonitis, colangitis, queratitis, miositis, hepatitis, encefalitis, nefritis e infección diseminada $^{18-26}$.

Además, se han comunicado infecciones por Encephalitozoon spp. en pacientes receptores de trasplantes de órganos sólidos (riñón, hígado y pulmón) y de precursores hematopoyéticos ${ }^{25,27-30}$.

Las microsporidiosis del tracto gastrointestinal (E. bieneusi y E. intestinalis) infectan el epitelio del tubo digestivo (intestino delgado y de las vías biliares). Encephalitozoon bieneusi además, puede causar una hepatitis granulomatosa, colestistitis crónica y colangitis esclerosante en pacientes con infección por VIH/SIDA.

Encephalitozoon spp. llega al intestino delgado afectando con mayor intensidad a la porción distal del duodeno e ileon proximal. Puede ser identificado en el ileon y, con menor frecuencia, en el colon. El microorganismo se localiza en la superficie apical del enterocito del intestino delgado y en las células epiteliales del tracto biliar y páncreas. Encephalitozoon intestinalis tiene capacidad invasora; sus esporas, en forma excepcional, logran alcanzar la superficie basal o bien la lámina propia, donde pueden acceder al torrente sanguíneo produciendo una diseminación visceral. Esta diseminación puede producir áreas de necrosis intestinal que clínicamente simulen un abdomen agudo.

La infección crónica se asocia a un síndrome de malabsorción intestinal por disminución del área de superficie mucosa e inmadurez funcional de las células epiteliales de la vellosidad intestinal ${ }^{12}$.

\section{Diagnóstico}

Para el diagnóstico a partir de muestras clínicas, el estándar de oro es la visualización del parásito en biopsias de intestino delgado mediante microscopia electrónica. 
Sin embargo, es una técnica invasora, costosa y que requiere personal entrenado ${ }^{12}$.

La visualización de las esporas de Microsporidium spp. en aguas, deposiciones, y otros fluidos, requiere métodos de concentración de la muestra. El sedimento concentrado se puede visualizar a través de microscopia de luz utilizando varios tipos de tinciones (cromotropo 2R, calcofluor, Kinyoun, Uvitex2B y Ziehl Neelsen).

La identificación de especie se realiza con anticuerpos monoclonales por IFI. Otros medios de diagnóstico son: cultivo celular, hibridación in situ, serología (ELISA, Western Blot) o biología molecular (RPC) $)^{31-33}$.

En nuestro centro se utilizan las tinciones de cromotropo 2R y Ziehl Neelsen, ambas con una sensibilidad y especificidad cercanas a $88 \%$. Pueden detectar todas las especies; sin embargo, no permite hacer diferenciación entre éstas. Recientemente, se incorporó la técnica de IFI con anticuerpos monoclonales, los que reaccionan exclusivamente con la pared de las esporas de Microsporidium E. bienusi $(1,3 \times 0,7 \mu \mathrm{m})$ y de E. intestinalis $(1,7 \times 1,0-1,1 \mu \mathrm{M})$, las especies más frecuentes tanto en pacientes inmunocomprometidos como en individuos inmunocompetentes. Éstas son marcadas en su superficie con fluorescencia periférica, logrando una sensibilidad cercana a $100 \%$ y especificidad de $88-90 \%$, con la ventaja de realizar el diagnóstico de especie. Tiene una lectura cualitativa (positiva o negativa). Este método diagnóstico, se encuentra validado y estandarizado, siendo el mismo que se utiliza en el ISP de Chile, centro de referencia nacional.

\section{Tratamiento}

La infección por Microsporidium spp. ocurre con mayor frecuencia en pacientes inmunocomprometidos siendo la recuperación del sistema inmune clave en la respuesta clínica en pacientes con microsporidiosis gastrointestinal, con eliminación del microorganismo y normalización de la arquitectura intestinal. En pacientes con infección por VIH/SIDA, parte del tratamiento primario es el inicio de TARV efectiva. Se han demostrado recaídas por falla en la administración de la terapia TARV asociada con la disminución de la función inmunológica y del recuento de linfocitos $\mathrm{CD} 4^{12}$.

Entre los fármacos ensayados in vitro e in vivo para el tratamiento de la microsporidiosis, tanto fumagilina como albendazol han demostrado tener eficacia clínica en humanos. Fumagilina se ha ensayado en receptores de trasplantes; sin embargo, se asocia a toxicidad hematológica (neutropenia, trombocitopenia) ${ }^{34-36}$. El mecanismo de acción de albendazol es la unión a la $\beta$-tubulina; es activo contra toda la familia Encephalitozonidae (E. cuniculi, $E$. intestinalis) in vitro, en concentraciones tan bajas como $0,1 \mathrm{mg} / \mathrm{mL}$; sin embargo, no es activo contra $E$. bieneusi $^{12}$. En la infección por E. intestinalis, albendazol ha demostrado ser efectivo en dosis de $400 \mathrm{mg}$, dos veces al día. Sin embargo, la duración de la terapia no está bien establecida, observándose recaídas al suspender el tratamiento. En pacientes inmunocomprometidos se recomienda mantener la terapia por un tiempo más prolongado (cuatro semanas) controlándose la función hepática, renal y hematológica. En personas con SIDA se recomienda continuar tratamiento hasta que el recuento de linfocitos CD4 sea $>200$ céls/ml o al menos hasta seis meses desde el inicio de la terapia anti-retroviral ${ }^{12,37}$.

Existen escasos reportes de estudios controlados de microsporidiosis causada por Encephalitozoon pero hay numerosos casos comunicados que que han demostrado eficacia de 2-4 semanas de albendazol $400 \mathrm{mg}$, dos veces al día (5-15 mg/kg/día, dosis máxima de $800 \mathrm{mg}$ / día). Este fármaco también ha tenido buena respuesta en pacientes con infección diseminada ${ }^{37}$. Los pacientes descritos recibieron albendazol por un mínimo de 21 días con buena respuesta clínica y logrando erradicación microbiológica.

\section{Prevención}

En el ambiente hospitalario, las esporas de E. cuniculi pueden sobrevivir y permanecer infectantes durante al menos un mes. Las esporas presentes en las superficies pueden inactivarse con una exposición por $30 \mathrm{~min}$ a la mayoría de los desinfectantes. Por esta razón, los procedimientos de limpieza y desinfección habituales de los hospitales son suficientes para limitar la infección ${ }^{12}$.

Sin embargo, el control de Microsporidium spp. en el agua es más difícil de lograr. Por esta razón, debe asegurarse medidas sanitarias que prevengan la contaminación del agua y alimentos. El cumplimiento de la higiene de manos y hábitos higiénicos apropiados, reducen la probabilidad de transmisión por contacto con conjuntiva y córnea. Debe aplicarse precauciones de contacto en pacientes con síndrome diarreico infectados con cualquier especie de Microsporidium.

Las estrategias para la prevención de infecciones y brotes nosocomiales por microorganismos presentes en el agua se basan en: la educación a los pacientes y familiares para minimizar la exposición; la mantención y limpieza de duchas y baños; medidas de mitigación durante las remodelaciones, construcciones y sistemas de desinfección y filtración. Recomendaciones específicas en unidades de pacientes inmunocomprometidos son el consumo directo de agua envasada o filtrada y sistemas de filtros en grifos y duchas ${ }^{1,7,9,11,38}$.

La importancia de la calidad del agua en los hospitales de nuestro país es un tema no resuelto, especialmente en áreas que atienden pacientes inmunocomprometidos. La infraestructura, en general, y los sistemas de almacenamiento y distribución de agua de nuestros hospitales son antiguos y los programas de limpieza y mantención 
no se realizan principalmente por falta de previsión y de recursos económicos.

El reporte de infecciones y brotes asociados al agua en recintos hospitalarios como el descrito, debe alertar a las autoridades hospitalarias, dar relevancia a este tema y destinar fondos a programas de limpieza y desinfección de sistemas de suministro de agua. Esto contribuirá, sin lugar a dudas, a mejorar la calidad y seguridad de la atención de nuestros pacientes inmunocomprometidos.

\section{Resumen}

Introducción: Los sistemas de suministro de agua potable de los hospitales constituyen un reservorio de una variedad de microorganismos potencialmente patógenos que pueden afectar especialmente a niños y pacientes inmunocomprometidos. Especies de Microsporidium spp. potencialmente patógenos para el hombre han sido identificadas en el agua potable. La microsporidiosis es una infección parasitaria oportunista en pacientes inmunocomprometidos. Objetivos y Método: Describir un brote de diarrea nosocomial por Microsporidium de la especie Encephalitozoon intestinalis. Resultados: Se registraron siete casos de diarrea por $E$. intestinalis, entre noviembre de 2012 y febrero de 2013, en una unidad de pacientes inmunocomprometidos del Hospital de Niños Luis Calvo Mackenna, comprobándose la presencia de Microsporidium spp. abundante en el agua potable y estanques del hospital. Los casos secundarios pudieron transmitirse por contacto. Las medidas de control fueron precauciones de contacto, no usar agua de grifos para lavado de manos, ingesta de agua envasada y desinfección de estanques. Conclusiones: Esta investigación corresponde a un brote nosocomial transmitido por agua potable. La importancia de la calidad del agua en los hospitales de nuestro país es un tema no resuelto, especialmente en áreas que atienden pacientes inmunocomprometidos. Debe asegurarse el cumplimiento de limpieza y desinfección de los sistemas de suministro de agua en los hospitales.

\section{Referencias bibliográficas}

1.- Centers for Disease Control and Prevention. Guidelines for environmental infection control in health-care facilities: recommendations of $\mathrm{CDC}$ and the Healthcare Infection Control Practices Advisory Committee (HICPAC) 2003: $1-235$.

2.- Rutala W, Weber D. Water as a reservoir for nosocomial pathogens. Infect Control Hosp Epidemiol 1997; 18:609-16.

3.- Squier C, Yu V L, Stout J E. Waterborne nosocomial infections. Current Infectious Disease Reports 2000; 2: 490-6.

4.- Williams M M, Armbruster C R, Arduino M J. Plumbing of hospital premises is a reservoir for opportunistically pathogenic microorganisms: a review. Biofouling 2013; 29 (2): 147-62.

5.- Anaissie E J, Penzak S R, Dignani M C. The hospital water supply as a source of nosocomial infections. Arch Intern Med 2002; 162: 1483-92.

6.- Merlani G M, Francioli P. Established and emerging waterborne nosocomial infections. Curr Opin Infect Dis 2003; 16: 343-7.

7.- Decker B K, Palmore T N. The role of water in healthcare-associated infections. Curr Opin Infect Dis 2013; 26: 345-51

8.- Leclerc H, Schwartzbrod L, Dei-Cas E. Microbial agents associated with waterborne diseases. Crit Rev Microbiol 2002; 28 : 371-409.

9.- Emmerson A M. Emerging waterborne infections in health-care settings. Emerg Infect Dis 2001; 7: 272-6.

10.- Mesquita-Rocha S, Godoy-Martínez P C, Gonçalves S S, Urrutia M D, Carlesse F,
Seber A, et al. The water supply system as a potential source of fungal infection in paediatric haematopoietic stem cell units. BMC Infect Dis 2013; 13: 289.

11.- Ferranti G, Marchesi I, Favale M, Borella P, Bargellini A. Aetiology, source and prevention of waterborne healthcare-associated infections: a review. J Med Microbiol 2014; 63 (Pt 10): 1247-59.

12.- Weiss L M. Capítulo 271: Microsporidiosis. En: Mandell, Douglas and Bennett's. Principles and Practice of Infectious Diseases. Mandell GL, Bennett JE, Dolin R. 7th edition. Ed. Churchill Livingstone Elsevier, Philadelphia 2010; p. 3391-407.

13.- Didier E S, Weiss L M. Microsporidiosis: Not just in AIDS patients. Curr Opin Infect Dis 2011; 24 (5): 490-5.

14.- Desportes I, Le Charpentier Y, Galian A, Bernard F, Cochand-Priollet B, Lavergne A, et al. Ocurrence of a new microsporidian: Enterocytozoon bieneusi sp., in the enterocytes of a human patient with AIDS. J Protozool 1985; 32: 250-4

15.- Dowd S E, Gerba C P, Pepper I L. Confirmation of the human-pathogenic Microsporidia Enterocytozoon bieneusi, Encephalitozoon intestinalis, and Vittaforma corneae in water. Appl Environ Microbiol 1998; 64: 3332-5.

16.- Enriquez F J, Taren D, Cruz-López A, Muramoto M, Palting J D, Cruz P. Prevalence of intestinal encephalitozoonosis in Mexico. Clin Infect Dis 1998; 26: 1227-9.

17.- Hutin Y J, Sombardier M N, Liguory O, Sarfati C, Derouin F, Modaï J, et al. Risk factors for intestinal microsporidiosis in patients with
HIV: Acase-control study. J Infect Dis 1998; 178: 904-7.

18.- Soule J B, Halverson A L, Becker R B, Pistole M C, Orenstein J M. A patient with AIDS and untreated Encephalitozoon intestinalis microsporidiosis leading to small bowel perforation: response to albendazole. Arch Pathol Lab Med 1997; 121: 880-7.

19.- Zender H O, Arrigoni E, Eckert J, Kapanci Y. A case of Encephalitozoon cuniculi peritonitis in a patient with AIDS. Am J Clin Pathol. 1989; 92: 352-6.

20.- Willson R, Harrington R, Stewart B, Fritsche T. Human immunodeficiency virus 1-associated necroziting cholangitis caused by infection with Septata intestinalis. Gastroenterology 1995; 108; 247-51.

21.- Rastrelli P, Didier E, Yee R. Microsporidial keratitis. Ophthalmol Clin North Am. 1994; 7 : 614-35.

22.- Coyle C M, Weiss L M, Rhodes L V 3rd, Cali A, Takvorian P M, Brown D F. Fatal myositis due to the microsporidian Brachiola algerae, a mosquito pathogen. $\mathrm{N}$ Engl J Med 2004; 351: 42-7.

23.- Terada S, Reddy K R, Jeffers L J, Cali A, Schiff E R. Microsporidan hepatitis in the acquired immunodeficiency syndrome. Ann Intern Med. 1987; 107: 61-2.

24.- Weber R, Deplazes P, Flepp M, Mathis A, Baumann R, Sauer B, et al. Cerebral microsporidiosis due to Encephalitozoon cuniculi in a patient with human immunodeficiency virus infection. N Engl J Med 1997; 336: 474-8

25.- Meissner EG, Bennett JE, Qvarnstrom Y, 
da Silva A, Chu EY, Tsokos M, et al. Disseminated microsporidiosis in an immunosuppressed patient. Emerg Infect Dis 2012; 18: 1155-8.

26.- Lanternier F, Boutboul D, Menotti J, Chandesris M O, Sarfati C, Mamzer Bruneel M F, et al. Microsporidiosis in solid organ transplant recipients: two Enterocytozoon bieneusi cases and review. Transpl Infect Dis 2009; 11: 83-8.

27.- Ladapo T A, Nourse P, Pillay K, Frean J, Birkhead M, Poonsamy B, et al. Microsporidiosis in pediatric renal transplant patients in Cape Town, South Africa: two case reports. Pediatr Transplant 2014; 18: E220-6.

28.- Agholi M, Hatam G R, Motazedian M H. Microsporidia and coccidia as causes of persistence diarrhea among liver transplant children: incidence rate and species/genotypes. Pediatr Infect Dis J 2013; 32:185-7.

29.- Levine D J, Riley D J, Jorgensen J H, McClain W D, Tio F, Visvesvara G S, et al. Key diagnostic features of granulomatous interstitial nephritis due to Encephalitozoon cuniculi in a lung transplant recipient. Am J Surg Pathol 2013; 37 (3): 447-52.

30.- Ambrosioni J, van Delden C, Krause K H,
Bouchuiguir-Wafa C, Nagy M, Passweg J, et al. Invasive microsporidiosis in allogeneic haematopoietic SCT recipients. Bone Marrow Transplant 2010; 45: 1249-51.

31.- Thellier M, Accoceberry I, Desportes I, Biligui S, Bart-Delabesse E, Ripert C, et al. Simple species diagnosis of human intestinal microsporidia by an immunofluorescence test using specific monoclonal antibodies: an evaluation study in two hospitals in France. First United Workshop on Microsporidia from Invertebrate and Vertebreate Hosts. Institute of Parasitology, Academy of Sciences of the Czech Republic, České Budějovice, Czech Republic, July 12-15, 2004. Disponible en: http://folia. paru.cas.cz/pdfs/fol/2005/01/01.pdf (acceso: 13 de octubre de 2015).

32.- Alfa Cisse O, Ouattara A, Thellier M, Accoceberry I, Biligui S, Minta D. Evaluation of an immunofluorescent-antibody test using monoclonal antibodies directed against Enterocytozoon bienusi and Encephalitozoon intestinalis for diagnosis of intestinal microsporidiosis in Bamako (Mali). J Clin Microbiol 2002; 40: 1715-8.

33.- Taniuchi M, Verweij J J, Sethabutr O, Bodhidatta L, García L, Maro A, et al. Multiplex polymerase chain reaction method to detect Cyclospora, Cystoisospora, and Microsporidia in stool samples. Diagn Microbiol Infect Dis 2011; 71: 386-90.

34.- Molina J M, Tourneur M, Sarfati C, Chevret S, de Gouvello A, Gobert J G, et al. Fumagillin treatment of intestinal microsporidiosis. N Engl J Med 2002; 346:1963-9.

35.- Champion L, Durrbach A, Lang P, Delahousse M, Chauvet C, Sarfati C, et al. Fumagillin for treatment of intestinal microsporidiosis in renal transplant recipients. Am J Transplant 2010; 10: 1925-30.

36.- Desoubeaux G, Maakaroun-Vermesse Z, Lier C, Bailly E, Morio F, Labarthe F, et al. Successful treatment with fumagillin of the first pediatric case of digestive microsporidiosis in a liverkidney transplant. Transpl Infect Dis. 2013; 15: E250-9.

37.- Gross U. Treatment of microsporidiosis including albendazole. Parasitol Res 2003; 90 Supp 1:S14-8.

38.- Exner M, Kramer A, Lajoie L, Gebel J, Engelhart S, Hartemann P. Prevention and control of health care-associated waterborne infections in health care facilities. Am J Infect Control 2005; 33 (5 Suppl 1): S26-40. 